\title{
Indicar estatinas a personas con riesgo cardiovascular global de $7,5 \%$ a diez años es costo-efectivo en los EE.UU.
}

\author{
Indicating statins for people with a global cardiovascular risk of 7,5\% at ten years is cost-effective in the USA
}

\section{Objetivos}

Comparar la costo-efectividad de indicar las estatinas en prevención primaria según diferentes umbrales de riesgo cardiovascular global a diez años. (RCVG).

\section{Diseño}

Análisis de costo-efectividad a través de un modelo de microsimulación, incluyendo un horizonte temporal de toda la vida, una perspectiva social y una tasa de descuento del $3 \%$ para los costos y los resultados en salud. Las fuentes de datos principales para los parámetros del modelo incluyeron encuestas nacionales de salud y nutrición y grandes ensayos clínicos o meta-análisis para los efectos de las estatinas.

\section{Lugar y pacientes}

Estados Unidos. Simulación de individuos de entre 40 y 75 años de características representativas del país.

\section{Intervenciones}

Estatinas en prevención primaria según diferentes umbrales de RCUG a diez años: desde no indicar estatinas en prevención

Pandya A y col. JAMA, 2015;314(2):142-150.

primaria hasta indicarlas a todos los pacientes.

\section{Medición de costos y resultados principales}

Se tomó en cuenta el costo de las estatinas, los costos en salud que ocasiona evaluar el RCVG, tanto en costos directos (ej. estudios de laboratorio), como indirectos (ej. horas de trabajo perdidas por los pacientes, costo del viaje a la consulta), el costo de los diferentes eventos cardiovasculares (ej. infarto, ictus) y los efectos adversos del tratamiento (ej. rabdomiolisis). Los beneficios se midieron en años de vida ajustados por la calidad (AVAC o QALY)

Además, se ajustó de acuerdo a los datos publicados sobre la adherencia al tratamiento.

\section{Resultados principales}

Los resultados principales se describen en la Tabla 1. Los resultados fueron altamente sensibles al precio de las estatinas, el grado de molestia que genera a los individuos tomar un comprimido diario y el riesgo de inducir diabetes mellitus.

\section{Conclusiones}

Tabla 1. Costo-efectividad de las estatinas según diferentes umbrales de riesgo cardiovascular global.

\begin{tabular}{c|c|c} 
Umbral de RCVG a 10 años & Costo en dólares de EE.UU. & RCEl\$ \\
\hline$\geq 20 \%$ & 21.898 & Dominio extendido* \\
\hline$\geq 10 \%$ & 22.455 & $30.000 /$ QALY \\
\hline$\geq 7,5 \%$ & 22.696 & $37.000 /$ QALY \\
\hline$\geq 3 \%$ & 23.406 & $140.000 /$ QALY \\
\hline$\geq 1 \%$ & 23.952 & Fuertemente dominada\# \\
\hline
\end{tabular}

RCVG: riesgo cardiovascular global. RCEl: razón de costo-efectividad incremental. $\ddagger$ La RCEl se interpreta como el costo adicional que se debe invertir para obtener un QALY adicional. ${ }^{*}$ Hay otras opciones más efectivas pero de peor costo-efectividad. \# Hay otras opciones más efectivas y de menor costo.

En adultos estadounidenses de 45 a 75 años, el umbral de RCVG a diez años mayor o igual a 7,5\% presenta una costo-efectividad aceptable para la indicación de estatinas, más claro al umbral mayor o igual a $10 \%$ y dominante con riesgo mayor o igual a
$20 \%$. El umbral era sensible a las preferencias del paciente para tomar una medicación diaria, cambios en el precio de estatina, y el riesgo de diabetes inducida por estatinas.

\section{Comentario}

Al ser este un estudio de microsimulación, los resultados que arroja son tan robustos como las fuentes de donde se extrajeron o las asunciones que se tomaron. La pregunta que se debe plantear es si los resultados de dichas fuentes estadounidenses son extrapolables a la población de nuestro país. Si bien esto es difícil de corroborar, ya que no contamos con datos tan exactos, la transferabilidad de los estudios de costo-efectividad siempre es limitada en ámbitos sanitarios y de países tan distintos. Aunque el estudio muestra que la administración de estatinas es costo-efectiva en su medio para los valores umbrales de RCVG a 10 años propuestos por las guías de los EE.UU. ${ }^{1}$ y el Reino Unido ${ }^{2}$ (7,5\% y $10 \%$ respectivamente), descono- cemos el punto de corte en nuestro medio y las guías americanas podrían sobreestimar el riesgo ${ }^{3}$. Estas consideraciones dan cuenta de las escasas herramientas con las que se cuenta para la toma de decisiones políticas en salud, desde datos poblacionales fidedignos, hasta saber cuánto es la voluntad de pago por QALY en cada región.

Conclusiones del comentador

En la práctica clínica cotidiana, la decisión de la medicación con estatinas debe ser individualizada, teniendo en cuenta las preferencias de cada paciente en el marco de la toma de decisiones compartidas.

Nahuel A. Orcaizaguirre. [ Servicio de Medicina Familiar y Comunitaria del Hospital Italiano de Buenos Aires. nahuel.orcaizaguirre@hospitalitaliano.org.ar ]

Orcaizaguirre NA. Indicar estatinas a personas con riesgo cardiovascular global de 7,5\% a diez años es costo-efectivo en EE.UU. Evid Act Pract Ambul. 2017;20(2):54. Comentado de: Pandya A y col. Cost-effectiveness of 10-Year Risk Thresholds for Initiation of Statin Therapy for Primary Prevention of Cardiovascular Disease. JAMA. 2015;314(2):142-150. PMID: 26172894.

\section{Referencias bibliográficas}

1. Stone NJ, y col. American College of Cardiology/American Heart Association Task Force on Practice Guidelines. ACC/AHA guideline on the treatment of blood cholesterol to reduce atherosclerotic cardiovascular risk in adults: a report of the American College of Cardiology/American Heart Association Task Force on Practice Guidelines. Circulation. 2014;129(25)(suppl 2):S1-S45

2. NICE. Lipid modification: cardiovascular risk assessment and the modification of blood lipids for the primary and secondary prevention of cardiovascular disease (CG181). 2014 Disponible en: https://www.nice.org.uk/guidance/cg181

3. McCarthy M. Use of controversial ACC/AHA guidelines for statin therapy is supported by US studies. BMJ. 2015 Jul 19;351:h3902. doi: 10.1136/bmj.h3902. 